\title{
Inflammatory bowel disease patients are frequently nonadherent to scheduled induction and maintenance infliximab therapy: A Canadian cohort study
}

\author{
Christopher Ma MD*, Chad J Evaschesen MD FRCPC*, Grenvil Gracias MD, Vivian W Huang MD FRCPC, \\ Darryl K Fedorak BSc, Karen I Kroeker MD FRCPC, Levinus A Dieleman MD PhD, \\ Brendan P Halloran MD FRCPC, Richard N Fedorak MD FRCPC
}

\begin{abstract}
C Ma, CJ Evaschesen, G Gracias, et al. Inflammatory bowel disease patients are frequently nonadherent to scheduled induction and maintenance infliximab therapy: A Canadian cohort study. Can J Gastroenterol Hepatol 2015;29(6):309-314.
\end{abstract}

BACKGROUND: Adherence to maintenance medication regimens in inflammatory bowel disease patients has traditionally been poor. Although infliximab has demonstrated efficacy in inducing and maintaining disease remission, adherence to regularly scheduled infliximab infusions is required to maintain therapeutic trough drug levels and prevent the development of anti-infliximab antibodies.

OBJECTIVES: To characterize patient adherence to regularly scheduled induction and maintenance infliximab infusions.

METHODS: A retrospective cohort study was conducted evaluating adult outpatients with Crohn disease or ulcerative colitis on an induction or maintenance regimen of regularly scheduled infliximab from 2008 to 2010 at the University of Alberta (Edmonton, Alberta). Nonadherence was defined by a discrepancy of $>72 \mathrm{~h}$ between the scheduled date of infusion and the actual date of administration. Patients were defined as nonadherent if they received $<80 \%$ of their infliximab infusions per schedule.

RESULTS: A total of 215 patients (173 Crohn disease, 42 ulcerative colitis) met the inclusion criteria. Patients received a median of $12.0 \mathrm{inf}$ liximab infusions (interquartile range 7.0 to 13.0 ) during the study period; 412 induction and 1837 maintenance infliximab infusions were administered. Of 140 patients, 109 (77.9\%) were adherent to their infliximab induction regimen, while 68 of 215 (31.6\%) were adherent to their infliximab maintenance regimen. One hundred ninety-eight of $215(92.1 \%)$ patients received at least one delayed maintenance infliximab infusion and $20(10.1 \%)$ received maintenance infusions, on average, $>1$ week late.

CONCLUSIONS: While three-quarters of patients are adherent to infliximab induction therapy, fewer than one-third remained adherent to their scheduled maintenance infliximab regimen.

Key Words: Adherence; Crohn disease; Inflammatory bowel disease; Infliximab; Ulcerative colitis

Crohn disease (CD) and ulcerative colitis (UC) are chronic relapsing and remitting inflammatory bowel diseases (IBD). Similar to many chronic illnesses, lifelong therapy is typically required to maintain patients in remission. However, adherence to medical therapy in this cohort has traditionally been quite poor; a systematic review involving 4322 IBD patients found widely varying rates of nonadherence to maintenance medication regimens, including some studies reporting nonadherence rates as high as $72 \%(1)$. Multiple variables contribute to poor adherence, including illness-,
Souvent, les patients atteints d'une maladie inflammatoire de l'intestin ne respectent pas le traitement d'induction et d'entretien prévu à l'infliximab : une étude de cohorte canadienne

HISTORIQUE : En général, les patients atteints d'une maladie inflammatoire de l'intestin respectent peu leur traitement médicamenteux. Même si l'efficacité de l'infliximab à induire et maintenir une rémission est démontrée, il faut adhérer aux perfusions régulières d'infliximab afin de maintenir les taux thérapeutiques minimaux et de prévenir l'apparition d'anticorps anti-infliximab.

OBJECTIF : Caractériser l'adhérence des patients à des perfusions régulières d'induction et d'entretien à l'infliximab.

MÉTHODOLOGIE : Entre 2008 et 2010, des chercheurs de l'université de l'Alberta, à Edmonton, ont mené une étude de cohorte rétrospective pour évaluer des patients ambulatoires adultes atteints de la maladie de Crohn ou de la colite ulcéreuse suivant un traitement régulier d'induction ou d'entretien à l'infliximab. La non-adhérence au traitement était définie par un écart de plus de 72 heures entre la date de perfusion prévue et la date d'administration. Les patients étaient définis comme n'adhérant pas au traitement s'ils recevaient moins de $80 \%$ de leurs perfusions d'infliximab par série.

RÉSULTATS : Au total, 215 patients (173 atteints de la maladie de Crohn, 42 de la colite ulcéreuse) respectaient les critères d'inclusion. Ils ont reçu une médiane de 12,0 perfusions d'infliximab (plage interquartile de 7,0 à 13,0 ) pendant la période de l'étude, soit 412 inductions et 1837 traitements d'entretien. Des 140 patients, 109 (77,9\%) adhéraient au traitement d'induction à l'infliximab, et 68 sur 215 (31,6\%), au traitement d'entretien. Cependant, 198 des 215 patients (92,1\%) ont tardé avant de recevoir au moins une perfusion d'entretien à l'infliximab, dont $20(10,1 \%)$ avaient en moyenne une semaine de retard.

CONCLUSIONS : Les trois quarts des patients adhèrent au traitement d'induction à l'infliximab, mais moins du tiers continue d'adhérer au traitement d'entretien prévu.

\footnotetext{
*Authors who contributed equally to the work

Division of Gastroenterology, University of Alberta, Edmonton, Alberta

Correspondence: Dr Richard N Fedorak, University of Alberta, 2-14A Zeidler Building, Edmonton, Alberta T6G 2X8. Telephone 780-492-6941, fax 780-492-8121, e-mail richard.fedorak@ualberta.ca

Received for publication March 24, 2015. Accepted May 9, 2015
} 
UC (6), its 'real-life' clinical effectiveness may be hampered by poor adherence. Specifically, secondary loss of response during maintenance therapy is a common phenomenon encountered in more than onehalf of IBD patients receiving anti-TNF agents (7), with development of antidrug antibodies playing a central role. In patients treated with infliximab, missing or delaying regularly scheduled infusions may contribute to drug immunogenicity (8), decreased serum trough drug levels (9) and, ultimately, adverse clinical outcomes. In fact, frequently missing or delaying infliximab infusions can mimic an episodic administration regimen, which compared with fixed scheduled infusion protocols in subgroup analysis of the A Crohn's Disease Clinical Trial Evaluating Infliximab in a New Long-Term Treatment Regimen (ACCENT I) trial resulted in increased likelihood of antidrug antibody development (28\% versus $9 \%$ ), reduced likelihood of mucosal healing ( $18 \%$ versus $44 \%$ ), increased hospitalizations (38\% versus $23 \%$ ), and reduced clinical response and remission rates (10).

Several authors have evaluated adherence to infliximab therapy in patients with IBD. Recently, Lopez et al (11) reviewed 13 studies evaluating adherence in this cohort and demonstrated varying rates of adherence ranging from $36 \%$ to $96 \%$. Nevertheless, there are several limitations to the results from these studies. Most findings are extrapolated from pharmacy refill data or administrative databases (12), medical insurance claims (13) or self-reported questionnaires (14), which are inherently subject to under-reporting, recall bias and coding inaccuracy. Furthermore, they may not reflect true medication adherence because these types of data do not capture administration of delayed or early infusions. Defining nonadherence is also particularly challenging and highly heterogeneous: previous authors have used the number of missed or delayed infusions, missing refill prescriptions, medication possession ratio $<80 \%$ or inadequate total infusions over a defined time period; however, these are all surrogate measures for assessing medication adherence.

Thus, in the present study, we assessed the 'true' adherence to regularly scheduled infliximab induction and maintenance therapy using manually searched medical chart review of scheduled and administered infliximab infusions. We define the proportion of patients who were nonadherent to infliximab therapy, characterize the incidence of early and delayed infusions and describe the variance around scheduled infusions. In a secondary analysis, we examined risk factors predicting nonadherence to long-term maintenance infliximab therapy.

\section{Study design and setting}

\section{METHODS}

The present retrospective cohort study was performed using data collected from $\mathrm{CD}$ and $\mathrm{UC}$ outpatients receiving maintenance infliximab therapy from August 2008 to August 2010, at the University of Alberta (Edmonton, Alberta). Patients were identified from the Division of Gastroenterology inflammatory bowel disease electronic database.

\section{Patient population}

Patients were eligible for inclusion if they met the following criteria: adult (>17 years of age); diagnosis of $\mathrm{CD}$ or UC; receiving either initial induction regimen of infliximab $5 \mathrm{mg} / \mathrm{kg}$ at weeks 0,2 and 6; or advanced onto a regularly scheduled maintenance outpatient infliximab regimen. Disease phenotype, infliximab dose escalation, concurrent medical therapy and duration of infliximab before the study were not contraindications to inclusion.

\section{Outcomes and definitions}

The primary objectives of the present study were to: determine the proportion of IBD patients who are adherent to a regularly scheduled infliximab infusion protocol; and to characterize the incidence of early and delayed infliximab administration. The secondary objective was to assess risk factors predicting infliximab nonadherence.

For the present study, nonadherence to a single infliximab infusion was defined as a discrepancy of $>72 \mathrm{~h}$ between the scheduled date of infliximab infusion and the actual date of administered infusion. There is no previous widely accepted definition for adherence to an individual infliximab infusion - a $72 \mathrm{~h}$ cut-off was decided on because this creates a six-day window for the patient to receive their infusion per protocol (three days before and three days after their scheduled infusion date). It was agreed that this was a sufficient time frame to accommodate statutory holidays, infusion clinic availability and minor personal reasons that may briefly delay or expedite the infusion schedule. A missed infliximab infusion was defined during maintenance therapy if the delay between administered infusions was $>12$ weeks.

Overall patient nonadherence was defined if $<80 \%$ of infliximab infusions were received adherently. Thus, patients requiring a temporary delay in infliximab infusions due to infection, perianal disease, holidays, etc, were not deemed to be nonadherent to therapy. This cut-off was based on previous studies involving IBD patients in which definitions of nonadherence included medication possession ratio $<80 \%$ and receiving $<7$ infliximab infusions in first year of therapy (seven infusions represents approximately $80 \%$ of the potential infusions for the first year of therapy, including three induction and six maintenance infusions) $(15,16)$.

The scheduled date of infliximab infusion was determined by the patient's optimal infliximab infusion regimen (ie, the next scheduled infusion would be eight weeks from the previous administered infusion if the patients is receiving maintenance therapy every eight weeks). For induction therapy, the scheduled infusion dates were at weeks 0,2 and 6. For maintenance therapy, the scheduled infusion dates were every eight weeks from the previous infusion. For patients requiring dose escalation during maintenance therapy and receiving infusions more frequently than every eight weeks, the scheduled date of infusion was adjusted accordingly.

Study follow-up time was defined from the date of first administered infusion to the date of last infusion received during the study inclusion period; total duration of infliximab therapy was determined from the date of first infliximab induction infusion.

\section{Data collection}

Data were extracted from official infliximab infusion records by two of the authors (CJE and GG) using a standardized case report form. These records included baseline patient demographic data, lifetime infliximab infusions received, infusion frequency (induction versus maintenance, maintenance infusion every eight weeks versus escalated/de-escalated schedule), actual date of administered infusion and previous medication exposure. Data were reviewed by two of the authors (CM and RNF).

Baseline patient data collected included sex, age, IBD diagnosis (CD versus UC), previous medical therapy (mesalamine or 5-aminosalicylate products, azathioprine or methotrexate) and total lifetime infliximab infusions received before inclusion. Subsequently, the scheduled infusion date was identified as described above and actual date of administered infliximab using the official infusion record. Clinical outcomes, including disease flares, hospital admissions and IBDrelated surgeries, were not available in the infusion records (these outcomes were outside the scope of the present study).

\section{Statistical methods}

For continuous variables, mean, SD, median and interquartile range (IQR) were calculated. The variance in days between date of actual administered and date of scheduled infusion was calculated for each infusion.

A post hoc sensitivity analysis was performed. Because there is no consensus definition of nonadherence to an individual infliximab infusion, adherence to maintenance infliximab therapy was determined if the definition of adherence was relaxed to allow a discrepancy between scheduled and administered infusion of $>7$ days (from three days). A sensitivity analysis was also performed whereby patients receiving $<6$ maintenance infusions were excluded; this enhanced the power of the analysis to better account for temporary causes of nonadherence.

Univariate and multivariate logistic regression analyses were performed to examine the association between patient variables and 
TABLE 1

Baseline patient demographics of $\mathbf{2 1 5}$ inflammatory bowel disease patients managed with induction or maintenance infliximab at the University of Alberta Inflammatory Bowel Disease Consultation and Research Clinic (Edmonton, Alberta) between 2008 and 2010

\begin{tabular}{ll}
\hline Characteristic & \\
\hline Inflammatory bowel disease diagnosis & $173(80.5)$ \\
Crohn disease & $42(19.5)$ \\
Ulcerative colitis & \\
Infliximab dosing & $140(65.1)$ \\
$\quad$ Induction dosing & $215(100.0)$ \\
$\quad$ Maintenance dosing & $144(67.0)$ \\
Infusion escalation during maintenance therapy & \\
Sex & $120(55.8)$ \\
$\quad$ Male & $95(44.2)$ \\
Female & \\
Age, years, mean \pm SD & $41.4 \pm 13.7$ \\
At study inclusion & $40.8 \pm 13.7$ \\
At infliximab induction & \\
Previous medications & $209(97.2)$ \\
$\quad$ Mesalamine or 5-ASA products & $197(91.6)$ \\
Immunomodulators (azathioprine or methotrexate) & \\
Infliximab Infusions, median (IQR) & $12.0(7.0-19.5)$ \\
Lifetime infusions & $12.0(7.0-13.0)$ \\
Infusions during the study period & \\
Follow-up, weeks, median (IQR) & $80.1(38.7-100.9)$ \\
Study follow-up duration & $80.1(38.7-138.7)$ \\
\hline Total time on infliximab &
\end{tabular}

Data presented as $n$ (\%) unless otherwise indicated. ASA Aminosalicylate; $I Q R$ Interquartile range

nonadherence, with results expressed as OR with 95\% CI. Age (defined as $>40$ and $\leq 40$ years), sex, IBD diagnosis (CD versus UC), previous medication exposure, requirement for infusion escalation and lifetime infliximab infusions (defined as $\leq 7$ infusions, eight to 12 infusions and $\geq 13$ infusions) were a priori included in the regression model. Fistulizing disease was not included in the logistic regression model because it would not apply to patients with UC.

Statistical analysis was performed using SPSS version 22.0 (IBM Corporation, USA).

Baseline patient characteristics

\section{RESULTS}

Patient demographics are summarized in Table 1. A total of 215 patients (173 CD, $42 \mathrm{UC})$ met the inclusion criteria. One hundred forty $(65.1 \%)$ patients received infliximab induction dosing during the study period and all patients received at least one maintenance infusion of infliximab. Patients received a median of 12.0 infliximab infusions (IQR 7.0 to 19.5) lifetime and were followed for a median duration of 80.1 weeks (IQR 38.7 to 100.9 weeks) during the study.

Forty-one (23.7\%) CD patients had fistulizing disease; 144 patients (67.0\%) required dose escalation of infliximab to more frequent infusions than every eight weeks (escalations to maintenance infusion every four to seven weeks, depending on the clinical circumstances and clinician judgement). Almost all patients had previous exposure to mesalamine, azathioprine or methotrexate.

Adherence with infliximab induction therapy

Adherence to infliximab induction infusions $(5 \mathrm{mg} / \mathrm{kg}$ at weeks 0,2 and 6) is summarized in Table 2. One hundred forty patients received a mean $( \pm \mathrm{SD})$ of $2.9 \pm 0.3$ infliximab induction infusions. A total of 412 infliximab induction infusions were administered.
TABLE 2

Patient adherence* to infliximab (IFX) induction therapy and characteristics of early and delayed IFX induction infusions in 140 inflammatory bowel disease patients

\begin{tabular}{lc}
\hline Variable & \\
\hline IFX induction, $\mathrm{n}$ & 140 \\
$\quad$ Patients receiving IFX induction therapy & 412 \\
$\quad$ Total administered IFX induction infusions & \\
Adherence to induction IFX & $109(77.9)$ \\
$\quad$ Patients adherent to IFX induction, $\mathrm{n}(\%)$ & $2.7 \pm 0.6$ \\
$\quad$ Adherent IFX induction infusions per patient, mean \pm SD & \\
Delayed induction infusions & $65(46.4)$ \\
$\quad$ Patients receiving at least one delayed IFX infusion, n (\%) & $0.5 \pm 0.6$ \\
$\quad$ Late infusions per patient, mean \pm SD & $1.5 \pm 1.8$ \\
$\quad$ Late days per infusion, mean \pm SD & $2.1 \pm 4.1$ \\
$\quad$ Cumulative late days per patient, mean \pm SD & \\
Early induction infusions & $67(47.9)$ \\
$\quad$ Patients receiving at least one early IFX infusion, $\mathrm{n}(\%)$ & $0.5 \pm 0.6$ \\
$\quad$ Early infusions per patient, mean \pm SD & $1.2 \pm 1.6$ \\
$\quad$ Early days per infusion, mean \pm SD & $1.1 \pm 2.8$ \\
$\quad$ Cumulative early days per patient, mean \pm SD & \\
\hline${ }^{*}$ Defined as receiving $>80 \%$ of induction infusions within 72 h of the regularly \\
scheduled date of infusion at weeks O, 2 and 6
\end{tabular}

\section{TABLE 3}

Patient adherence* to infliximab (IFX) maintenance therapy and characteristics of early and delayed IFX maintenance infusions in $\mathbf{2 1 5}$ inflammatory bowel disease patients

\begin{tabular}{|c|c|}
\hline \multicolumn{2}{|l|}{ IFX maintenance } \\
\hline Patients receiving IFX maintenance therapy, $\mathrm{n}$ & 215 \\
\hline IFX maintenance infusions, median (IQR) & $9.0(4.0-13.0)$ \\
\hline Total administered IFX maintenance infusions, $\mathrm{n}$ & 1837 \\
\hline \multicolumn{2}{|l|}{ Adherence to maintenance IFX } \\
\hline Patients adherent to IFX maintenance, $\mathrm{n}(\%)$ & $68(31.6)$ \\
\hline Adherent IFX maintenance infusions per patient, mean \pm SD & $6.0 \pm 3.8$ \\
\hline Total missed IFX infusions ${ }^{\dagger}, \mathrm{n}(\%)$ & $32(1.7)$ \\
\hline \multicolumn{2}{|l|}{ Delayed maintenance infusions } \\
\hline Patients receiving at least one delayed IFX infusion, $\mathrm{n}(\%)$ & $198(92.1)$ \\
\hline Late infusions per patient, mean \pm SD & $3.5 \pm 2.5$ \\
\hline Late days per infusion, days, mean $\pm \mathrm{SD}$ & $3.3 \pm 4.6$ \\
\hline Patients delaying infusions, on average, $>7$ days, $n(\%)$ & $20(10.1)$ \\
\hline \multicolumn{2}{|l|}{ Early maintenance infusions } \\
\hline Patients receiving at least one early IFX infusion, $\mathrm{n}(\%)$ & $171(79.5)$ \\
\hline Early infusions per patient, mean \pm SD & $2.1 \pm 1.8$ \\
\hline Early days per infusion, days, mean \pm SD & $1.2 \pm 1.6$ \\
\hline
\end{tabular}

${ }^{*}$ Patient adherence defined as receiving $>80 \%$ of maintenance infusions within $72 h$ of regularly scheduled date of infusion; ${ }^{\dagger} A$ maintenance infliximab infusion was considered to be missed if the delay between administered infusions was $>12$ weeks

Of 140 patients, $109(77.9 \%)$ were adherent to their infliximab induction infusions and received, on average, $2.7 \pm 0.6$ induction infusions with adherence. Sixty-five $(46.4 \%)$ patients received at least one delayed induction infusion, but mean delay time was short (1.5 \pm 1.8 days). A similar number of patients received at least one early infliximab induction infusion $(\mathrm{n}=67$ [47.9\%]).

Adherence to infliximab maintenance therapy

Adherence to infliximab maintenance therapy is summarized in Table 3. Two hundred fifteen patients received a total of 1837 infliximab maintenance infusions. Over the two-year study period, patients received a median of 9.0 maintenance infusions (IQR 4.0 to 13.0).

Sixty-eight of $215(31.6 \%)$ patients were adherent to $>80 \%$ of their infliximab maintenance infusions. On average, patients were adherent 
to $6.0 \pm 3.8$ maintenance infliximab infusions over the follow-up period and, on average, accumulated $25.8 \pm 35.5$ late days. Only 32 maintenance infliximab infusions were completely missed; however, nearly all (92.1\% [198 of 215]) patients received at least one delayed maintenance infusion. Although the mean delay per infusion was $3.3 \pm 4$.6 days, $10 \%$ (20 of 198) of patients received delayed infusions, on average, $>1$ week late.

Mean variance from the scheduled infliximab maintenance infusion was $4.0 \pm 4.6$ days. As such, a patient would be expected to accrue a dropped infliximab maintenance infusion every 13.9 infusions (112.0 weeks) based on the standard maintenance regimen of infliximab infusion every eight weeks. Among the 20 patients experiencing a mean delay in administration of infliximab $>7$ days, a dropped infliximab maintenance infusion would be accrued every 33.3 weeks.

\section{Sensitivity analysis}

Exclusion of patients receiving $<6$ maintenance infusions in sensitivity analysis did not change overall adherence to therapy: only 46 of $145(31.7 \%)$ patients in this cohort were adherent to their maintenance regimen. When the definition of nonadherence to an individual infliximab infusion was relaxed to allow a discrepancy between scheduled and administered infusion date of $>7$ days, 101 of $215(46.5 \%)$ patients were adherent to $>80 \%$ of their infliximab maintenance infusions.

\section{Predictors of nonadherence during maintenance therapy}

Univariate and multivariate ORs for risk factors predicting nonadherence to infliximab maintenance therapy are summarized in Appendix 1. Only male sex was associated with slightly increased risk for nonadherence in both univariate (OR 1.73 [95\% CI 1.00 to 2.99]; $\mathrm{P}=0.049$ ) and multivariate (OR 1.77 [95\% CI 1.01 to 3.11]; $\mathrm{P}=0.046$ ) regression analysis. No other factors reached statistical significance.

\section{DISCUSSION}

Infliximab has a central role in the management of patients with moderate-to-severe IBD and, although it has demonstrated efficacy in randomized clinical trials, real-life clinical effectiveness may be limited by poor adherence. Previous studies evaluating anti-TNF therapy compliance in this cohort have primarily used administrative data and surrogate markers of adherence such as medication possession ratio (15). While these measures capture substantial lapses in adherence, they lack the resolution to identify nonadherence on an infusion-to-infusion basis. Here, we present a large Canadian retrospective evaluation of 215 IBD patients treated with infliximab, and assess through detailed chart review, the true adherence to both induction and maintenance infliximab infusion regimens. We found that while $77.9 \%$ of patients were adherent to induction infusions, fewer than one-third of patients had sustained infusion adherence and $10 \%$ will experience delayed infusions, on average, $>1$ week from the ideal scheduled dosing protocol during maintenance therapy.

Adherence to infliximab was first examined by Kane et al (12) in 2006; they described $96 \%$ adherence to therapy after retrospectively reviewing 1185 infusions using administrative and pharmacy refill data. This incredibly high adherence rate relates to the use of 'no show' visits as the definition for nonadherence. Similarly, we found a very low incidence of completely missed infusions (1.7\%). Other authors, using administrative database definitions for nonadherence including medication possession ratio $<80 \%$ or $<7$ infusions during the first year of treatment, have also found varying rates of adherence, ranging from $57.1 \%$ to $79.8 \%(13,14,16)$.

In contrast to previous studies in which nonadherence was defined only by completely missed infusions, we believe that frequently delayed infliximab infusions are a reflection of poor patient compliance with the treatment regimen. Thus, we used a generous definition of adherence to both individual infusions (within six days of scheduled infusion) as well as overall adherence ( $80 \%$ of infusions received per schedule) to describe patient adherence to infliximab therapy with greater resolution. Other authors have used similarly constructed composite definitions of nonadherence (17). Our definition for nonadherence accounts for common barriers to compliance including patient inconvenience or statutory holidays (patients can schedule their infusion for the next available business day if the scheduled infusion date falls on a weekend or holiday and still be considered adherent to therapy) as well as unpredictable or excusable reasons for delaying infliximab infusions such as personal emergencies, infections or drug adverse effects.

Overall, adherence to anti-TNF therapy, given its necessity for scheduled intravenous administrations, may be slightly better compared with adherence to oral maintenance therapies including 5-aminosalicylate products and immunomodulators. A systematic review of oral therapy compliance in IBD patients by Jackson et al (1) found that most studies report approximately 30\% to $45 \%$ nonadherence rates. This disparity may reflect differences in disease activity (highly active disease requiring biologic therapy may prompt better adherence), route and schedule of administration (intravenous versus oral, every eight week maintenance versus daily therapy), and more frequent clinician follow-up and monitoring. However, it should be highlighted that even among patients on infliximab therapy, there exists a significant rate of nonadherence. Exploring reasons for nonadherence was outside the scope of the present study but has been evaluated elsewhere (18).

Few studies have directly evaluated delays to individual scheduled infliximab infusions and are predominantly reported in abstract form. In a small retrospective cohort of 82 IBD patients on infliximab, Duncan et al (19) found that infusions were postponed in 14 of 82 (17\%) patients over 12-month follow-up. Similarly, another abstract by Angelucci et al (20) found that $19.1 \%$ of infusions were delayed, median delayed administrations per patient was three infusions, and the median delay was 9.6 days (range one to 35 days). Both authors cite 'technical' reasons, including patient forgetfulness, inconvenience and intentional nonadministration as the reason for delayed infusion in $>90 \%$ of cases. In our cohort, we report even higher rates of delayed infusions, delayed infusions per patient and total accumulated late days, most likely related to our increased sample size and duration of follow-up. Specific indication for delayed infliximab administration is not part of our infliximab infusion record. However, medically appropriate indications for delaying infusions, such as adverse events or infections, are accounted for by our definition of nonadherence. These specific indications for delaying infusions would presumably only affect a minority of the patient's total infusions and, thus, they should still be able to receive $>80 \%$ of their infusions with adherence. In fact, Angelucci et al (20) report that only 3.3\% of nonadherent infusions were due to adverse events in their cohort.

Delays and nonadherence with infliximab therapy may have significant implications on clinical outcomes in patients with IBD. In particular, we hypothesize that nonadherence has a substantial impact on long-term maintenance of clinical response and secondary loss of response. In both prospective (10) and retrospective (21) cohorts, it has been shown that patients managed with regularly scheduled infliximab compared with episodically administered therapy have improved outcomes for mucosal healing, disease-related hospitalizations and surgeries, and maintained clinical response. While all patients in our cohort were on scheduled therapy, prolonged and recurrent delays to infusion can mimic a more episodic pattern of administration. Unfortunately, clinical outcomes, including hospitalizations, surgeries and disease flares, are not tracked in our infliximab infusion records. Thus, we are unable to correlate infliximab adherence with disease outcomes, although this should be evaluated in future studies.

The mechanism behind primary and secondary loss of response to infliximab is influenced by subtherapeutic serum trough drug levels predisposing to the formation of neutralizing anti-infliximab antibodies (22). Recently, in a post hoc analysis of the ACCENT trial, 
Cornillie et al (23) found that CD patients with week-14 infliximab trough level $\geq 3.5 \mu \mathrm{g} / \mathrm{mL}$ were 3.5 times more likely to achieve durable sustained response through week 54 of therapy (OR 3.5 [95\% CI 1.1 to 11.4). Similar evidence exists in UC, in which detectable serum infliximab drug levels are significantly associated with clinical remission and decreased risk for colectomy (24). We hypothesize that delayed and nonadherent infliximab infusions increase the time during which patients have subtherapeutic infliximab trough levels and are predisposed to forming antidrug antibodies. Therapeutic drug monitoring with infliximab trough levels and antibody prevalence was not clinically available during the time of the study in our jurisdiction. Future studies should evaluate the relationship between delays in administered infliximab, therapeutic drug monitoring and, ultimately, clinical outcomes.

Nonadherence to infliximab maintenance therapy also has significant implications on health care utilization (25). Using the Integrated Health Care Information Service claims database, Kane et al (16) found significantly increased health care utilization costs among nonadherent CD patients on infliximab, who were 2.5 times more likely to require CD-related hospitalization, had 90\% greater CD-related medical costs and $115 \%$ greater hospitalization costs compared with infliximab-adherent patients. Wan et al (26) estimated treatment costs using propensity-weighted regression models and reported similar results. Although nonadherent patients had expectedly reduced infliximab drug costs $(\$ 14,889$ versus $\$ 28,289 ; \mathrm{P}<0.001)$, they had substantially increased costs for hospitalizations $(\$ 17,634$ versus $\$ 2,458$; $\mathrm{P}<0.001)$, outpatient visits $(\$ 10,909$ versus $\$ 7,357 ; \mathrm{P}<0.001)$ and emergency room visits ( $\$ 458$ versus $\$ 236 ; \mathrm{P}<0.001)$.

Early infusions did occur in our cohort, although this was less common and patients accumulated fewer early days compared with delayed infusions (6.9 early versus 25.8 late days). Although earlier infusion is likely preferable to delayed infusion to ensure ongoing maintenance of clinical response, excessively early administration may result in elevated infliximab trough levels, which have been shown to be associated with adverse events including dermatological reactions (27).

Definitive predictors of infliximab adherence have not been consistently reported across multiple studies $(11,28)$. Kane et al $(12)$ previously found that female patients were more likely to be nonadherent to infliximab but interestingly, the opposite trend was observed in our cohort, and nonadherent behaviours have been well described in male patients by other authors $(29,30)$. Whether this relates to differences in patient population, financial drug coverage, temporal trends in infliximab use or sampling error is unclear. Kane et al (12) also reported that patients were more likely to be nonadherent for an infusion $>18$ weeks after induction; although not statistically significant in our regression analysis, the higher rate of adherence observed during induction therapy in our cohort compared with maintenance therapy suggests attrition of patient adherence behaviours over time. Additionally, we found that requirement for infusion escalation trended toward increased nonadherence; we hypothesize that common 'technical' reasons for delayed infusions such as forgetfulness and inconvenience are magnified in patients requiring more frequent infliximab infusions.

There were several limitations to the present study. First, although detailed review of infusion records enabled us to analyze infliximab drug adherence with more resolution than previous studies, we were limited in collecting other clinical parameters, including disease phenotype (particularly for CD), severity of disease and disease activity, clinical response and remission status, concurrent medical therapy and noninvasive biomarkers of inflammation (eg, C-reactive protein). This limited the power of our regression analysis for identifying predictors of nonadherence. A second limitation was that reasons for nonadherence were not available based on the infusion record. Although previous authors have defined patient-related 'technical' factors as being the predominant reason for nonadherence, we are unable to confirm this here. Medical reasons for delaying infliximab infusions such as opportunistic infections could not be captured in our data set, but we have attempted to minimize this potential misclassification bias using a composite definition of nonadherence as described above. While the criterion for defining adherence to an individual infliximab infusion may appear strict (within $72 \mathrm{~h}$ of schedule), this actually creates a six-day window for the patient to receive their infusion per protocol. Even when the definition for adherence was extended to seven days in the sensitivity analysis, fewer than onehalf of patients were adherent.

Third, data are available to 2010 but may be less generalizable because experience with infliximab has evolved. Additionally, there may be referral bias because the present study was conducted at a tertiary care centre. In less specialized community gastroenterology practices, adherence to therapy may be even worse, particularly if infusion sites are not centralized, potentially amplifying the effects of preventable reasons associated with poor compliance (eg, inconvenience).

Finally, although we recognize that therapeutic drug monitoring will become a critical component of IBD management moving forward, data regarding serum infliximab trough levels and antibodies to infliximab were not available in our jurisdiction at the time of the study. Furthermore, clinical outcomes relevant to patients with IBD, including hospitalization, surgery and disease flare, are not recorded in our infliximab infusion database. These are major outcomes of interest associated with anti-TNF therapy adherence but were outside the scope of the present study and should be evaluated in future studies.

\section{CONCLUSION}

Although three-quarters of IBD patients were adherent to infliximab induction therapy, only one-third sustained adherence during maintenance regimens. Frequently, patients on infliximab experience delays to infusion, and future studies should evaluate whether these delays result in altered drug immunogenicity, increased antiinfliximab antibody formation or adverse patient outcomes.

DISCLOSURES: RNF has served as a consultant and advisory board member for AbbVie Canada Inc and Janssen Canada Inc, and received clinical/basic research grants from AbbVie and Janssen. KIK, LAD and $\mathrm{BPH}$ have served as consultants and speakers for AbbVie and Janssen. The remaining authors have no financial disclosures or conflicts of interest to declare.

FUNDING: This study was funded, in part, by the Alberta Innovates Health Solutions, Alberta Inflammatory Bowel Disease Consortium, and the Centre of Excellence for Gastrointestinal Inflammation and Immunity Research (CEGIIR).

AUTHOR CONTRIBUTIONS: RNF is acting as the article guarantor and contributed to study design, data collection, data analysis and manuscript editing. CM contributed to data analysis, manuscript drafting, and editing. CJE contributed to study design, data collection and data analysis. GG contributed to data collection and data analysis. VH, DKF, $\mathrm{KIK}, \mathrm{LAD}$ and BPH contributed to manuscript editing. All authors have approved the final version of the manuscript, including the authorship list. 


\begin{tabular}{|c|c|c|}
\hline \multirow{2}{*}{$\begin{array}{l}\text { Risk factor predicting non- } \\
\text { adherence to maintenance } \\
\text { infliximab therapy }\end{array}$} & \multicolumn{2}{|c|}{ OR $(95 \% \mathrm{Cl})$} \\
\hline & Univariate & Multivariate \\
\hline \multicolumn{3}{|l|}{ IBD diagnosis } \\
\hline Crohn disease & 1.00 & 1.00 \\
\hline Ulcerative colitis & $0.74(0.37-1.46)$ & $0.62(0.29-1.29)$ \\
\hline Male sex & $1.73(1.00-2.99)$ & $1.77(1.01-3.11)$ \\
\hline Age $>40$ years & $1.43(0.77-2.66)$ & $1.37(0.71-2.64)$ \\
\hline $\begin{array}{l}\text { Previous mesalamine/5-ASA } \\
\text { exposure }\end{array}$ & $0.87(0.17-4.39)$ & $0.64(0.11-3.54)$ \\
\hline $\begin{array}{l}\text { Previous azathioprine or } \\
\text { methotrexate }\end{array}$ & $0.67(0.26-1.78)$ & $0.64(0.22-1.84)$ \\
\hline $\begin{array}{l}\text { Requirement for infliximab } \\
\text { infusion escalation }\end{array}$ & $1.54(0.86-2.74)$ & $1.40(0.67-2.94)$ \\
\hline \multicolumn{3}{|l|}{ Lifetime infliximab infusions } \\
\hline$\leq 7$ & 1.00 & 1.00 \\
\hline $8-12$ & $1.52(0.71-3.26)$ & $1.44(0.65-3.15)$ \\
\hline$\geq 13$ & $0.81(0.43-1.53)$ & $0.93(0.43-2.04)$ \\
\hline
\end{tabular}

${ }^{*}$ Nonadherence defined as receiving $<80 \%$ of infliximab maintenance infusions within $72 \mathrm{~h}$ of scheduled date. ASA Aminosalicylate

\section{REFERENCES}

1. Jackson CA, Clatworthy J, Robinson A, Horne R. Factors associated with non-adherence to oral medication for inflammatory bowel disease: A systematic review. Am J Gastroenterol 2010;105:525-39.

2. Lopez-Sanroman A, Bermejo F. Review article: How to control and improve adherence to therapy in inflammatory bowel disease. Aliment Pharmacol Ther 2006;(24 Suppl 3):45-9.

3. Kane S, Huo D, Aikens J, Hanauer S. Medication nonadherence and the outcomes of patients with quiescent ulcerative colitis. Am J Med 2003;114:39-43.

4. Hanauer SB, Feagan BG, Lichtenstein GR, et al. Maintenance infliximab for Crohn's disease: The ACCENT I randomised trial. Lancet 2002;359:1541-9.

5. Sands BE, Anderson FH, Bernstein CN, et al. Infliximab maintenance therapy for fistulizing Crohn's disease. N Engl J Med 2004:350:876-85.

6. Rutgeerts P, Sandborn WJ, Feagan BG, et al. Infliximab for induction and maintenance therapy for ulcerative colitis. N Engl J Med 2005;353:2462-76.

7. Ma C, Huang V, Fedorak DK, et al. Crohn's disease outpatients treated with adalimumab have an earlier secondary loss of response and requirement for dose escalation compared to infliximab: A real life cohort study. J Crohn's Colitis 2014;8:1454-63.

8. Hanauer SB, Wagner CL, Bala M, et al. Incidence and importance of antibody responses to infliximab after maintenance or episodic treatment in Crohn's disease. Clin Gastroenterol Hepatol 2004:2:542-53

9. Maser EA, Villela R, Silverberg MS, Greenberg GR. Association of trough serum infliximab to clinical outcome after scheduled maintenance treatment for Crohn's disease. Clin Gastroenterol Hepatol 2006;4:1248-54.

10. Rutgeerts P, Feagan BG, Lichtenstein GR, et al. Comparison of scheduled and episodic treatment strategies of infliximab in Crohn's disease. Gastroenterology 2004;126:402-13.
11. Lopez A, Billioud V, Peyrin-Biroulet C, Peyrin-Biroulet L. Adherence to anti-TNF therapy in inflammatory bowel diseases: A systematic review. Inflamm Bowel Dis 2013;19:1528-33.

12. Kane S, Dixon L. Adherence rates with infliximab therapy in Crohn's disease. Aliment Pharmacol Ther 2006;24:1099-103.

13. Carter CT, Leher H, Smith P, Smith DB, Waters HC. Impact of persistence with infliximab on hospitalizations in ulcerative colitis. Am J Manag Care 2011;17:385-92.

14. Kitney L, Turner JM, Spady D, et al. Predictors of medication adherence in pediatric inflammatory bowel disease patients at the Stollery Children's Hospital. Can J Gastroenterol 2009;23:811-5.

15. Carter CT, Waters HC, Smith DB. Effect of a continuous measure of adherence with infliximab maintenance treatment on inpatient outcomes in Crohn's disease. Patient Prefer Adherence 2012;6:417-26.

16. Kane SV, Chao J, Mulani PM. Adherence to infliximab maintenance therapy and health care utilization and costs by Crohn's disease patients. Adv Ther 2009;26:936-46.

17. Arranz EM, Arranz MDM, Herranz MJ, et al. Compliance to the scheduled treatment with infliximab. Experience in a tertiary care hospital. J Crohn's Colitis 2012;6:S156.

18. Horne R, Parham R, Driscoll R, Robinson A. Patients' attitudes to medicines and adherence to maintenance treatment in inflammatory bowel disease. Inflamm Bowel Dis 2009;15:837-44.

19. Duncan J, Sastrillo M, Baker J, et al. An audit of adherence to antiTNF therapy in patients with inflammatory bowel disease. Gut 2011;60:A141-A2.

20. Angelucci E, Galletti B, De Nitto D, et al. Adherence to antitumor necrosis factor alpha therapy in inflammatory bowel diseases: An Italian monocentric experience. Gut 2011;60:A410.

21. Stein DJ, Ananthakrishnan AN, Issa M, et al. Impact of prior irregular infliximab dosing on performance of long-term infliximab maintenance therapy in Crohn's disease. Inflamm Bowel Dis 2010;16:1173-9.

22. Ben-Horin S, Chowers Y. Loss of response to anti-TNF treatments in Crohn's disease. Aliment Pharmacol Ther 2011;33:987-95.

23. Cornillie F, Hanauer SB, Diamond RH, et al. Postinduction serum infliximab trough level and decrease of $\mathrm{C}$-reactive protein level are associated with durable sustained response to infliximab: A retrospective analysis of the ACCENT I trial. Gut 2014;63:1721-7.

24. Seow CH, Newman A, Irwin SP, Steinhart AH, Silverberg MS, Greenberg GR. Trough serum infliximab: A predictive factor of clinical outcome for infliximab treatment in acute ulcerative colitis. Gut 2010;59:49-54.

25. Carter CT, Waters HC, Smith DB. Impact of infliximab adherence on Crohn's disease-related healthcare utilization and inpatient costs. AdvTher 2011;28:671-83.

26. Wan GJ, Kozma CM, Slaton TL, Olson WH, Feagan BG. Inflammatory bowel disease: healthcare costs for patients who are adherent or non-adherent with infliximab therapy. J Med Economics 2014;17:384-93

27. Huang V, Dhami N, Fedorak DK, et al. Infliximab trough levels are correlated with infliximab-associated adverse events. Gastroenterology 2014;146:S-1.

28. Fidder HH, Singendonk MM, van der Have M, Oldenburg B, van Oijen MG. Low rates of adherence for tumor necrosis factoralpha inhibitors in Crohn's disease and rheumatoid arthritis: Results of a systematic review. World J Gastroenterol 2013;19:4344-50.

29. Sewitch MJ, Abrahamowicz M, Barkun A, et al. Patient nonadherence to medication in inflammatory bowel disease. Am J Gastroenterol 2003;98:1535-44.

30. Kaplan GG, Panaccione R, Hubbard JN, et al. Inflammatory bowel disease patients who leave hospital against medical advice: Predictors and temporal trends. Inflamm Bowel Dis 2009;15:845-51. 


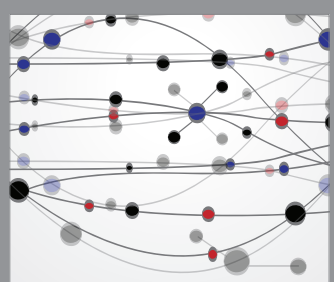

The Scientific World Journal
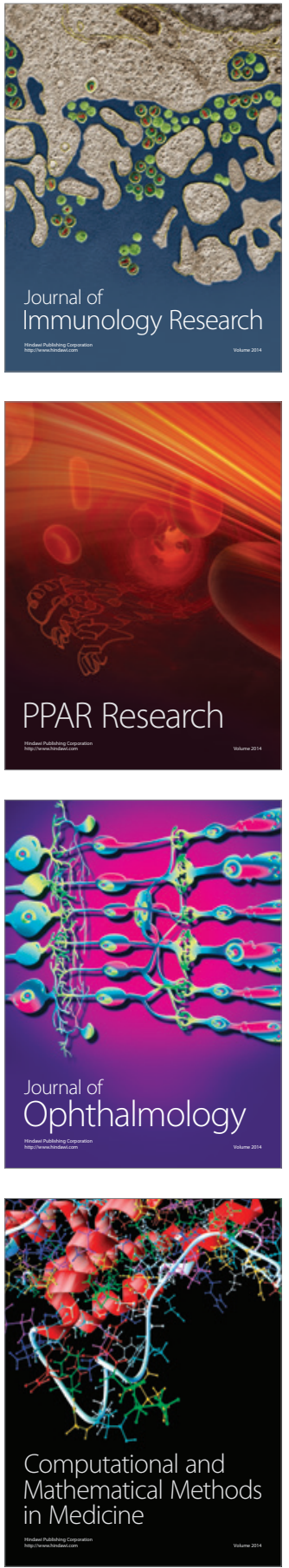

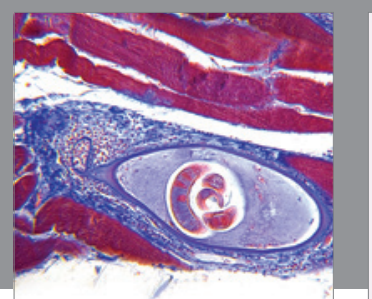

Gastroenterology Research and Practice

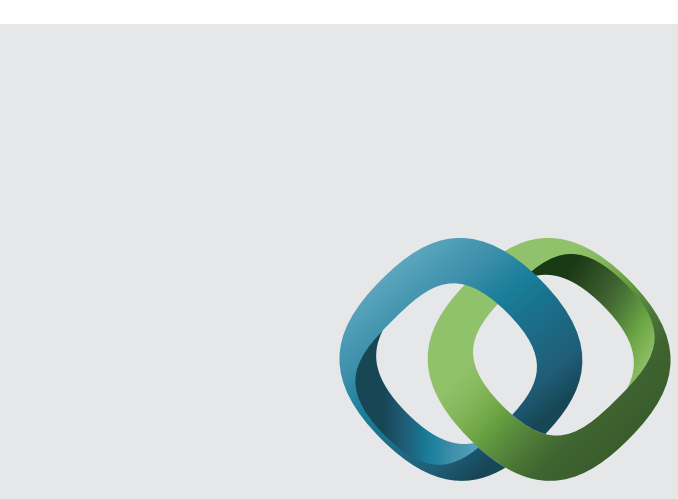

\section{Hindawi}

Submit your manuscripts at

http://www.hindawi.com
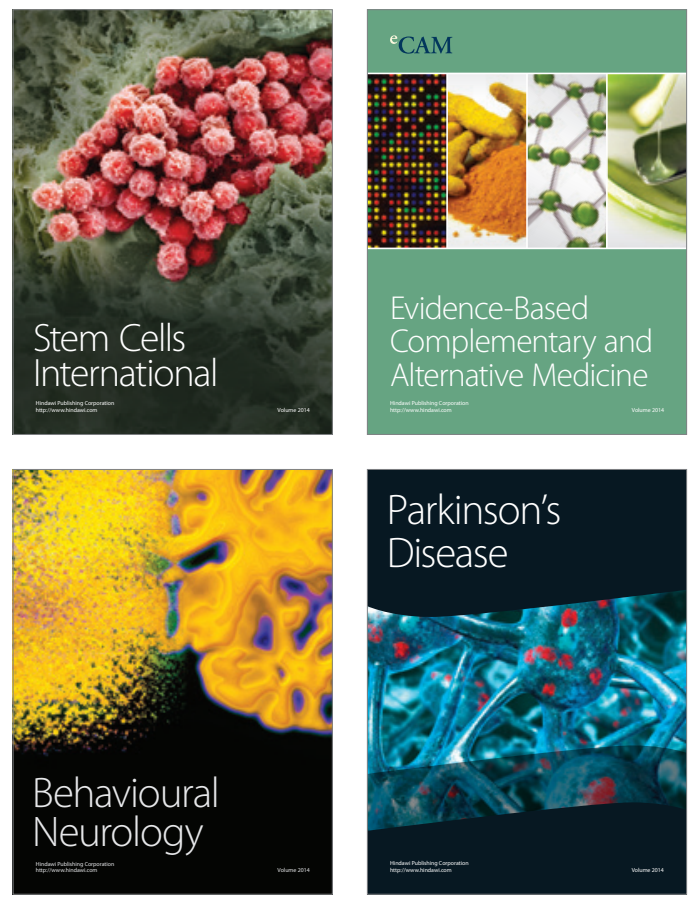
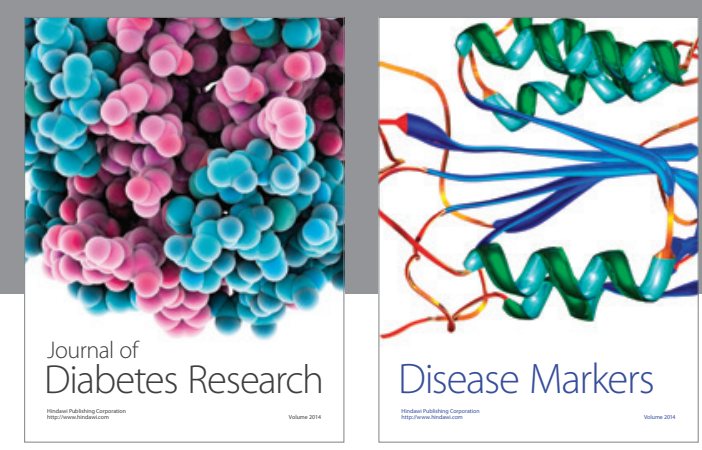

Disease Markers
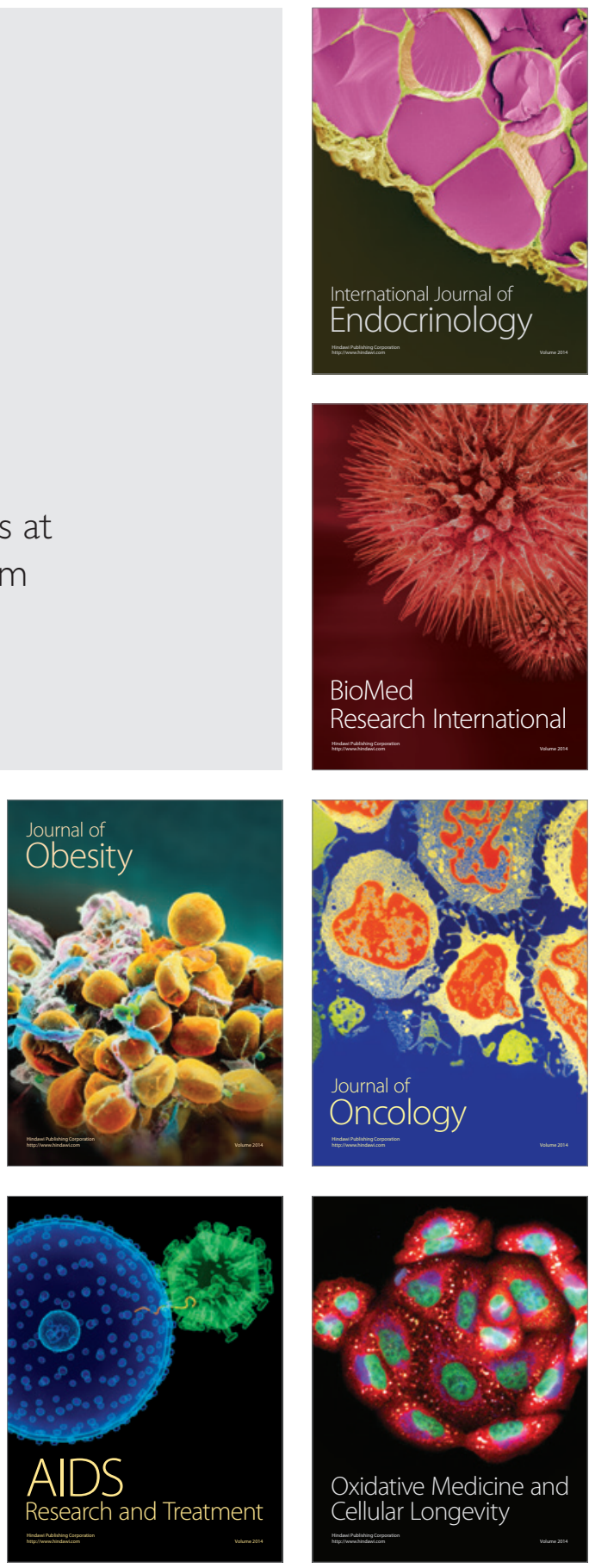